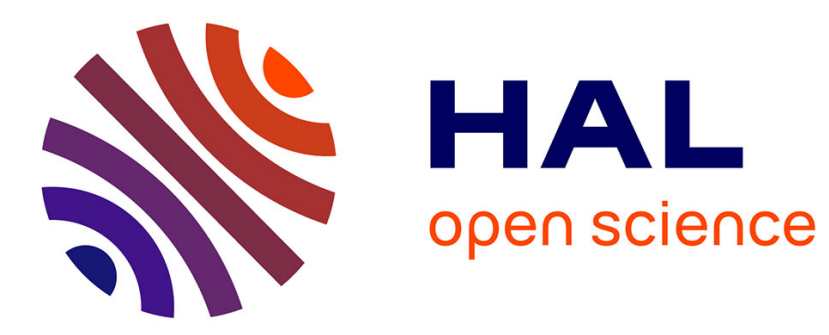

\title{
Robustness of an Adaptive Output Feedback for an Anti-Damped Boundary Wave PDE in Presence of In-Domain Viscous Damping
}

Christophe Roman, Delphine Bresch-Pietri, Christophe Prieur, Olivier Sename

\section{- To cite this version:}

Christophe Roman, Delphine Bresch-Pietri, Christophe Prieur, Olivier Sename. Robustness of an Adaptive Output Feedback for an Anti-Damped Boundary Wave PDE in Presence of In-Domain Viscous Damping. ACC 2016 - American Control Conference, Jul 2016, Boston, MA, United States. hal-01349352

\section{HAL Id: hal-01349352 \\ https://hal.science/hal-01349352}

Submitted on 27 Jul 2016

HAL is a multi-disciplinary open access archive for the deposit and dissemination of scientific research documents, whether they are published or not. The documents may come from teaching and research institutions in France or abroad, or from public or private research centers.
L'archive ouverte pluridisciplinaire HAL, est destinée au dépôt et à la diffusion de documents scientifiques de niveau recherche, publiés ou non, émanant des établissements d'enseignement et de recherche français ou étrangers, des laboratoires publics ou privés. 


\title{
Robustness of an Adaptive Output Feedback for an Anti-Damped Boundary Wave PDE in Presence of In-Domain Viscous Damping
}

\author{
Christophe Roman ${ }^{1}$, Delphine Bresch-Pietri ${ }^{2}$, Christophe Prieur $^{2}$ and Olivier Sename ${ }^{3}$
}

\begin{abstract}
This paper presents a robustness result for the use of a previously developed adaptive output-feedback controller designed for a pure wave PDE. This one-dimensional wave PDE has a boundary actuation opposite to an unknown anti-damping dynamics boundary. We prove that stabilization is still valid in the case of in-domain viscous damping using a controller designed neglecting the damping, provided that the damping coefficient is small enough. Our robustness proof grounds on the use of an alternative pure wave PDE, neglecting the indomain damping. We compare these two systems and apply a tailored backstepping transformation to carry out Lyapunov analysis. Numerical simulations show the relevance of our result for drilling applications and illustrate the merits of this control design.
\end{abstract}

\section{INTRODUCTION}

Numerous systems in various physical areas are modeled by wave equations, from sound propagation and creation to the generation of vibrations in mechanical structures [13]. Even if the underlying propagation phenomenon is marginally stable, some of those systems exhibit unstable behaviors. This is the case, e.g., of some engine combustion modes [1] and of drilling process in the oil industry [8]. For these two examples, instability arises from an anti-damped effect occurring at one of the system boundaries.

In this paper, we consider a linear wave equation subject to in-domain viscous damping with a boundary actuation opposite to an unknown anti-damping dynamics boundary. This modeling is representative of the aforementioned cases of study (see Section V later). Even if one may expect indomain damping to have a stabilizing effect, its appearance significantly complexifies the control task by adding an extra coupling. While several output feedback laws exist in the nominal case without damping [15], [3], [9], up to our knowledge, there exists only one design accounting distributed damping [16]. However, this solution, which grounds on the backstepping methodologies [10], is a full state feedback. This, for practical application, requires the development of an observer, which is still an open question.

In this paper, we propose to apply the adaptive boundary control law designed in [3], which, in addition to its only use of boundary measurements, counteracts uncertainties on the anti-damping coefficients. Introducing Riemann variables, the plant is reformulated as two coupled transport PDEs (with

\footnotetext{
${ }^{1}$ C. Roman is with Univ. Grenoble Alpes, GIPSA-lab, 11 rue des Mathématiques, 38000 Grenoble, France (christophe.roman@gipsa-lab.fr)

${ }^{2}$ D. Bresch-Pietri and C. Prieur are with CNRS, GIPSA-lab, 11 rue des Mathématiques, 38000 Grenoble, France

${ }^{3} \mathrm{O}$. Sename is with Grenoble INP, GIPSA-lab, 11 rue des Mathématiques, 38000 Grenoble, France
}

opposite directions) also coupled with an Ordinary Differential equation (ODE). Comparing this system to an estimated one neglecting damping, we prove closed-loop stability and convergence, provided that the damping coefficient is small enough. This robustness result is the main contribution of the paper.

The paper is organized as follows. In Section II, we present the problem under consideration before stating our main result in Section III, which contains also a discussion on a Riemann change of variable. Section IV is devoted to the proof of the main result. Finally some simulations to illustrate the behavior of our problem on a oil-inspired drilling application are presented in Section V. We conclude with directions of future work.

Notation In this paper, $|\cdot|$ is the Euclidean norm and $\|u(\cdot)\|$ is the spatial $L_{2}$-norm of a functional $[0,1] \ni x \mapsto u(x, \cdot)$, which is denoted as

$$
\|u(\cdot)\|=\sqrt{\int_{0}^{1} u(x, \cdot)^{2} d x}
$$

Sometimes, when the context is clear, the abusive notation $\|u\|$, will be used to denote $\|u(\cdot)\|$.

For $(a, b) \in \mathbb{R}^{2}$ such that $a<b$, let us define the standard projector operator on the interval $[a, b]$ as a function of two scalar arguments $f$ (denoting the parameter being updated) and $g$ (denoting the nominal update law) in the following manner:

$$
\operatorname{PROJ}_{[a, b]}(f, g)=g\left\{\begin{array}{cc}
0 & \text { if } f=a \text { and } g<0 \\
0 & \text { if } f=b \text { and } g>0 \\
1 & \text { otherwise }
\end{array}\right.
$$

\section{PROBLEM STATEMENT}

Let us consider the following system of a wave equation with in-domain viscous damping, subject to an anti-damping boundary, with actuation on the opposite boundary

$$
\begin{aligned}
& u_{t t}(x, t)=u_{x x}(x, t)-2 \lambda u_{t}(x, t) \\
& u_{x}(1, t)=U(t) \\
& u_{t t}(0, t)=a q u_{t}(0, t)+a\left[u_{x}(0, t)-d\right]
\end{aligned}
$$

in which $U(t)$ is the scalar control input, $\left(u, u_{t}\right)$ is the system state, with $\left(u(., 0), u_{t}(., 0)\right) \in H_{1}(0,1) \times L_{2}(0,1), a>0$ is a scalar constant. The in-domain viscous damping coefficient is $\lambda \geqslant 0$. The anti-damping coefficient is $q>0$ and $d$ is the static bias term. These last two quantities are supposed to be unknown constant values. In addition to being uncertain, the plant has all its eigenvalues in the right-hand half 
complex plane. In-domain damping in (3) also makes the control design significantly harder by introducing an internal coupling, as appears in the following sections. Here, we propose to use the adaptive controller developed in [3] for the case $\lambda=0$ (and given below), and to study if the closed-loop convergence is still achieved for $\lambda>0$.

In indirect adaptive control, a certain assumption over the boundedness of the unknown parameter values is needed.

Assumption 1: There exist known constants $q, \bar{q}, \underline{d}$ and $\bar{d}$ such that $q<\bar{q}, \underline{d}<\bar{d}$ and $q \in[q, \bar{q}], d \in[\underline{d}, \bar{d}]$.

Consider the following control law, designed in [3]:

$$
\begin{array}{r}
U(t)=-u_{t}(1, t)+\hat{d}(t)-\left(c_{0}+\hat{q}(t)-1\right)\left(e^{a(\hat{q}(t)-1)} u_{t}(0, t)\right. \\
\left.+a \int_{t-1}^{t} e^{a(\hat{q}(t)-1)(t-\tau)}(\eta(\tau)-\hat{d}(t)) d \tau\right)
\end{array}
$$

in which $c_{0}>0$ is a constant, $\hat{q}(t)$ is an estimate of the unknown parameter $q, \hat{d}(t)$ is an estimate of $d$, and

$$
\eta(t)=U(t)+u_{t}(1, t)
$$

The parameter update laws are:

$$
\begin{aligned}
& \dot{\hat{q}}(t)=\frac{a \gamma_{q}}{1+N(t)} \operatorname{PROJ}_{[\underline{q}, \bar{q}]}\left\{\hat{q}(t), u_{t}(0, t)\left(u_{t}(0, t)\right.\right. \\
& \left.\left.+b_{1}\left(c_{0}+\hat{q}(t)-1\right) \int_{t-1}^{t} e^{(a(\hat{q}(t)-1)-1)(\tau-t+1)} \sigma(\tau, t) d \tau\right)\right\} \\
& \dot{\hat{d}}(t)=\frac{a \gamma_{d}}{1+N(t)} \operatorname{PROJ}_{[\underline{d}, \bar{d}]}\left\{\hat{d}(t),-u_{t}(0, t)\right. \\
& \left.-b_{1}\left(c_{0}+\hat{q}(t)-1\right) \int_{t-1}^{t} e^{(a(\hat{q}(t)-1)-1)(\tau-t+1)} \sigma(\tau, t) d \tau\right\} \\
& N(t)=u_{t}(0, t)^{2}+b_{1} \int_{t-1}^{t} e^{\tau-t+1} \sigma(\tau, t)^{2} d \tau \\
& +b_{2} \int_{t-1}^{t} e^{\tau-t+1}\left(2 u_{t}(0, \tau)-\eta(\tau-1)+\hat{d}(t)\right)^{2} d \tau
\end{aligned}
$$

in which the bounds $q, \bar{q}, \underline{d}, \bar{d}$ are defined in Assumption 1 , PROJ is defined in (2), $b_{1}, b_{2}>0$ are normalization constants, the update gains $\gamma_{d}, \gamma_{q}>0$ are tuning parameters and, for $t \geqslant 0$ and $t-1 \leqslant \tau \leqslant t$,

$$
\begin{aligned}
\sigma(\tau, t) & =\eta(\tau)-\hat{d}(t)+\left(c_{0}+\hat{q}(t)-1\right)\left(e^{a(\hat{q}(t)-1)(\tau-t+1)}\right. \\
& \left.\times u_{t}(0, t)+a \int_{t-1}^{\tau} e^{a(\hat{q}(t)-1)(\tau-\chi)}(\eta(\chi)-\hat{d}(t)) d \chi\right)
\end{aligned}
$$

As this controller was developed in [3] for $\lambda=0$, the paper objective is to study its applicability for the damped case. In particular, it seems necessary to evaluate for which range of in-domain damping coefficient values the closedloop convergence is obtained. This is formulated in the next section.

\section{ROBUSTNESS RESULT}

Theorem 1: Consider the closed-loop system consisting of the plant (3)-(5), the control law (6) and the parameter update laws (9)-(8). Under Assumption 1, define the functional $\Gamma(t)$

$$
\begin{aligned}
& \Gamma(t)=u_{t}(0, t)^{2}+\int_{0}^{1} u_{t}(x, t)^{2} d+\int_{0}^{1}\left(u_{x}(x, t)-d\right)^{2} d x \\
&+(q-\hat{q})^{2}+(d-\hat{d})^{2}
\end{aligned}
$$

For any $c_{0}>0$, there exists $\lambda^{*}>0$-depending on initial condition $\max _{s \in[0,2]} \Gamma(-s)$ - such that, choosing any $\lambda \in\left[0, \lambda^{*}\right]$, there exist positive constants $b_{2}\left(c_{0}, \lambda^{*}\right), b_{1}\left(c_{0}, b_{2}, \lambda^{*}\right)$, and $\gamma^{*}\left(c_{0}, b_{1}, b_{2}, \lambda^{*}\right)$ such that for all $\gamma_{d} \in\left(0, \gamma^{*}\right)$ and $\gamma_{q} \in\left(0, \gamma^{*}\right)$, then there are $R>0$ and $\rho>0$ such that

$$
\Gamma(t) \leqslant R\left(e^{\rho \max _{s \in[0,2]} \Gamma(-s)}-1\right), \quad \forall t \geqslant 0
$$

and the regulation in $L_{2}$-norm follows, i.e

$$
\begin{aligned}
\lim _{t \rightarrow \infty} u_{t}(0, t) & =\lim _{t \rightarrow \infty}\left\|u_{t}(t)\right\| \\
& =\lim _{t \rightarrow \infty}\left\|u_{x}(t)-d\right\|=\lim _{t \rightarrow \infty}(\hat{d}-d)=0
\end{aligned}
$$

This result can be easily interpreted in the light of the control design which was directly inspired from the undamped case. Note that the stability result (13) is expressed with a delayed function. This is due to the PDE coupling introduced by in-domain damping. Besides, this result is semi-global $\left(\lambda^{*}\right.$ depends on initial conditions) as a result of the normalization involved in (8) and (9).

\section{PROOF OF THEOREM 1}

The proof of Theorem 1 consists of the following steps. First we reformulate the plant under consideration into coupled first order PDEs using Riemann variables. Then, expressing the control and parameter update laws under spacial form, we apply a backstepping transformation. This enables us to perform a standard Lyaunov analysis as a third step. We conclude this proof by showing and expressing the stability result in terms of the variables of (3)-(5).

\section{A. Reformulation of the system in Riemann variables}

Using the following Riemann coordinates and notations

$$
\begin{aligned}
\zeta(x, t) & =u_{t}(x, t)+u_{x}(x, t)-\hat{d}(t) \\
\omega(x, t) & =u_{t}(x, t)-u_{x}(x, t)+\hat{d}(t) \\
W(t) & =u_{t}(1, t)+U(t)-\hat{d}(t) \\
v(t) & =u_{t}(0, t)
\end{aligned}
$$

the wave system (3)-(5) is inversibly rewritten into the following system, which is referred as the Original system,

$$
\begin{aligned}
\dot{v}(t) & =a(q-1) v(t)+a[\zeta(0, t)-\widetilde{d}] \\
\zeta_{t}(x, t) & =\zeta_{x}(x, t)-\dot{\hat{d}}(t)-\lambda(\zeta+\omega) \\
\zeta(1, t) & =W(t) \\
\omega_{t}(x, t) & =-\omega_{x}(x, t)+\dot{\hat{d}}(t)-\lambda(\zeta+\omega) \\
\omega(0, t) & =2 v(t)-\zeta(0, t)
\end{aligned}
$$

We have now one ODE and two first-order PDEs, which are transport phenomena with source terms, the first one (21) with actuation on one boundary (22), and driving the ODE (20) through its second boundary. The second transport phenomenon (23) is backward and driven by the state of the ODE $(v(t))$ and the boundary condition of the first PDE. We have two source terms in each transport equation due to the in-domain damping. If we consider the system without indomain damping $(\lambda=0)$, the system writes as a cascade of two subsystems and the ODE can be controlled applying time 
delay systems theory. This is the idea motivating the control law (6), which has been designed using predictor techniques for input-delay ODEs, e.g., [2], [12], and [11]. Moreover, the presence of in-domain damping $(\lambda \neq 0)$, acting as a distributed coupling law (21) and (23), breaks this cascade. Theorem 1 states that if the dependence can be neglected, namely, if the damping coefficient is small enough, the control law (6)-(9) achieves stability and convergence.

\section{B. Global reformulation of the system}

Let us rewrite the system (20)-(24) into two other systems called the Estimated and the Auxiliary ones. The Estimated system is a plant transformation, the dynamics of which are the same as the system without in-domain damping. To define these systems, let us first consider the following variables

$$
\begin{aligned}
\hat{\zeta}(x, t) & =\zeta(x, t)+\lambda \int_{x}^{1}(\zeta(s, t+x-s)+\omega(s, t+x-s)) d s \\
\hat{\omega}(x, t) & =\omega(x, t)+\lambda \int_{0}^{x}(\zeta(s, t-x+s)+\omega(s, t-x+s)) d s \\
& -\lambda \int_{0}^{1}(\zeta(s, t-x-s)+\omega(s, t-x-s)) d s
\end{aligned}
$$

This leads to

$$
\begin{aligned}
\hat{\zeta}_{t}(x, t) & =\zeta_{t}(x, t)+\lambda \int_{x}^{1}\left(\zeta_{t}(\chi, t+x-\chi)+\omega_{t}(\chi, t+x-\chi)\right) d \chi \\
\hat{\zeta}_{x}(x, t) & =\zeta_{x}(x, t)-\lambda(\zeta(x, t)+\omega(x, t)) \\
& +\lambda \int_{x}^{1}\left(\zeta_{t}(\chi, t+x-\chi)+\omega_{t}(\chi, t+x-\chi)\right) d \chi
\end{aligned}
$$

which satisfies

$$
\hat{\zeta}_{t}(x, t)-\hat{\zeta}_{x}(x, t)=-\dot{\hat{d}}(t)
$$

We have now a dynamics without $\lambda$ : the in-domain damping coupling. And similarly with (26), we compute

$$
\hat{\omega}_{t}(x, t)=-\hat{\omega}_{t}(x, t)+\dot{\hat{d}}(t)
$$

We have again a dynamics without $\lambda$ : the in-domain damping coupling. Let now compute the boundary condition. From (25), one gets

$$
\begin{aligned}
& \hat{\zeta}(0, t)=\zeta(0, t)+\lambda \int_{0}^{1}(\zeta(\chi, t-\chi)+\omega(\chi, t-\chi)) d \chi \\
& \hat{\zeta}(1, t)=W(t)=\zeta(1, t)
\end{aligned}
$$

and from (26), one gets

$$
\hat{\omega}(0, t)=\omega(0, t)-\lambda \int_{0}^{1}(\zeta(\chi, t-\chi)+\omega(\chi, t-\chi)) d \chi
$$

With (24) and (29), it follows

$$
\hat{\omega}(0, t)=2 v(t)-\hat{\zeta}(0, t)
$$

1) Estimated system: Equations (25) and (26) lead to the Estimated system, that is

$$
\left\{\begin{aligned}
\hat{\zeta}_{t}(x, t) & =\hat{\zeta}_{x}(x, t)-\dot{\hat{d}}(t) \\
\hat{\zeta}(1, t) & =W(t) \\
\hat{\omega}_{t}(x, t) & =-\hat{\omega}_{x}(x, t)+\dot{\hat{d}}(t) \\
\hat{\omega}(0, t) & =2 v(t)-\hat{\zeta}(0, t)
\end{aligned}\right.
$$

Note that it has the same form as the one studied in [3]. As the transformation which maps the Original system, (20)(24), into the Estimated system, (32) is not reversible, more information is needed.

2) Auxiliary system: Let us now consider the difference between the Estimated system and the Original one, i.e, we consider the dynamics of $\widetilde{\zeta}(x, t)=\zeta(x, t)-\hat{\zeta}(x, t)$ and $\widetilde{\omega}(x, t)=\omega(x, t)-\hat{\omega}(x, t)$. Some computations with the help of (21)-(24) give

$$
\begin{aligned}
& \widetilde{\zeta}_{t}(x, t)=\widetilde{\zeta}_{x}(x, t)-\lambda(\widetilde{\zeta}(x, t)+\widetilde{\omega}(x, t)+\hat{\zeta}(x, t)+\hat{\omega}(x, t)) \\
& \widetilde{\zeta}(1, t)=0 \\
& \widetilde{\omega}_{t}(x, t)=-\widetilde{\omega}_{x}(x, t)-\lambda(\widetilde{\zeta}(x, t)+\widetilde{\omega}(x, t)+\hat{\zeta}(x, t)+\hat{\omega}(x, t)) \\
& \widetilde{\omega}(0, t)=-\widetilde{\zeta}(0, t)
\end{aligned}
$$

Now rewrite the ODE (20) with variables $\widetilde{\zeta}(x, t)$ and $\hat{\zeta}(x, t)$,

$$
\dot{v}(t)=a(q-1) v(t)+a[\widetilde{\zeta}(0, t)+\hat{\zeta}(0, t)-\widetilde{d}(t)]
$$

The complete system consisting of the Estimated system, (32), the Auxiliary system, (33) together with the ODE (34) is the one considered from now on.

C. Rewriting of the control and adaptive laws, and backstepping transformation

1) Rewriting of the control and adaptive laws: The previous form of the control and adaptive laws is useful for implementation, but another form is needed for the analysis. This is the purpose of this section. The control law (6), (using the notation (18) and (19) and the change of variables under the integral $\tau=t+x-1$ ), can be rewritten as

$$
\begin{aligned}
W(t)= & -\left(c_{0}+\hat{q}(t)-1\right)\left(e^{a(\hat{q}(t)-1)} v(t)\right. \\
& \left.+a \int_{0}^{1} e^{a(\hat{q}(t)-1)(1-x)}[\eta(t+x-1)-\hat{d}(t)] d x\right)
\end{aligned}
$$

With (7) and (18), one gets

$$
\eta(\tau)=W(\tau)+\hat{d}(\tau)
$$

System (32) includes two transport phenomena with source term, they satisfy for $(x, y) \in[0,1]^{2}$

$$
\begin{aligned}
\hat{\zeta}(x, t) & =\hat{\zeta}(y, t+x-y)-\hat{d}(t)+\hat{d}(t+x-y) \\
\hat{\omega}(x, t) & =\hat{\omega}(y, t-x+y)+\hat{d}(t)-\hat{d}(t-x+y)
\end{aligned}
$$

Taking $y=1$ in (37), with the boundary condition of the first transport phenomenon (33), and matching (25), (35), and (36), one finally gets

$$
\begin{aligned}
W(t)=-\left(c_{0}+\hat{q}(t)\right. & -1)\left(e^{a(\hat{q}(t)-1)} v(t)\right. \\
& \left.+a \int_{0}^{1} e^{a(\hat{q}(t)-1)(1-x)} \hat{\zeta}(x, t) d x\right)
\end{aligned}
$$

This form allows us to write a backstepping transformation, which will put one boundary condition equal to zero, that is

$$
\begin{aligned}
\hat{z}(x, t) & =\hat{\zeta}(x, t)+\left(c_{0}+\hat{q}(t)-1\right)\left(e^{a(\hat{q}(t)-1) x} v(t)\right. \\
& \left.+a \int_{0}^{x} e^{a(\hat{q}(t)-1)(x-\chi)} \hat{\zeta}(\chi, t) d \chi\right)
\end{aligned}
$$


Similarly with (37) and (38), the adaptive laws can be rewritten as

$$
\begin{aligned}
\dot{\hat{q}}(t) & =\frac{a \gamma_{q}}{1+N(t)} \operatorname{PROJ}_{[\underline{q}, \bar{q}]}\{\hat{q}(t), y(t)(y(t) \\
& \left.\left.+b_{1}\left(c_{0}+\hat{q}(t)-1\right) \int_{0}^{1} e^{(a(\hat{q}(t)-1)-1) x} \hat{z}(x, t) d x\right)\right\} \\
\dot{\hat{d}}(t) & =\frac{a \gamma_{d}}{1+N(t)} \operatorname{PROJ}_{[\underline{d}, \bar{d}]}\{\hat{d}(t),-y(t) \\
& \left.-b_{1}\left(c_{0}+\hat{q}(t)-1\right) \int_{0}^{1} e^{(a(\hat{q}(t)-1)-1) x} \hat{z}(x, t) d x\right\} \\
N(t) & =y(t)^{2}+b_{1} \int_{0}^{1} e^{x}(\hat{z}(x, t))^{2} d x+b_{2} \int_{0}^{1} e^{1-x}(\hat{\omega}(x, t))^{2} d x
\end{aligned}
$$

This form will allow us to simplify the Lyapunov analysis.

2) Backstepping transformation and target system: Consider the previous backstepping transformation (40). Note that it has the same form as the one in [3], but on the Estimated variable, $\hat{\zeta}(x, t)$ now. The Estimated system and the ODE, i.e (33) and (34), can then be reformulated reversibly as the following target system,

$$
\begin{aligned}
& \dot{v}(t)=-c_{0} a v(t)+a[\hat{z}(0, t)+\widetilde{\zeta}(0, t)+v(t) \widetilde{q}(t)-\widetilde{d}(t)] \\
& \hat{z}_{t}(x, t)=\hat{z}_{x}(x, t)+\dot{\hat{q}} g_{q}(x, t)+\dot{\hat{d}}(t) g_{d}(x, t) \\
& \quad+[\widetilde{q}(t) v(t)-\widetilde{d}(t)+\widetilde{\zeta}(0, t)] h(x, t) \\
& \hat{z}(1, t)=0
\end{aligned}
$$

$$
\begin{aligned}
& \hat{\omega}_{t}(x, t)=-\hat{\omega}(x, t)+\dot{\hat{d}}(t) \\
& \hat{\omega}(0, t)=\left(c_{0}+\hat{q}(t)+1\right) v(t)-\hat{z}(0, t)
\end{aligned}
$$

where

$$
\begin{aligned}
g_{q}(x, t) & =e^{a(\hat{q}(t)-1) x} v(t)+a \int_{0}^{x} e^{a(\hat{q}(t)-1)(x-y)} \hat{\zeta}(y, t) d y \\
& +\left(c_{0}+\hat{q}(t)-1\right)\left(a x e^{(\hat{q}(t)-1) x} v(t)\right. \\
& \left.+a^{2} \int_{0}^{x}(x-y) e^{a(\hat{q}(t)-1)(x-y)} \hat{\zeta}(y, t) d y\right) \\
g_{d}(x, t) & =-1-\left(c_{0}+\hat{q}(t)-1\right) a \int_{0}^{x} e^{a(\hat{q}(t)-1)(x-y)} d y \\
h(x, t) & =a\left(c_{0}+\hat{q}(t)-1\right) e^{a(\hat{q}(t)-1) x}
\end{aligned}
$$

we proposed a reformulation of the global system, i.e (33) and (44)-(48), with the use of the inverse backstepping transformation in (33):

$$
\begin{aligned}
\hat{\zeta}(x, t) & =\hat{z}(x, t)-\left(c_{0}+\hat{q}(t)-1\right)\left(e^{-a c_{0} x} v(t)\right. \\
& \left.+a \int_{0}^{x} e^{-a c_{0}(x-\chi)} \hat{z}(\chi, t) d \chi\right)
\end{aligned}
$$

\section{Stability analysis}

1) Lyapunov analysis: Let us consider the following Lyapunov functional candidate:

$$
V(t)=V_{\text {estim }}(t)+V_{\text {damp }}(t)
$$

with

$$
\begin{aligned}
& V_{\text {estim }}(t)=\log (1+N(t))+\frac{\widetilde{q}(t)^{2}}{\gamma_{q}}+\frac{\widetilde{d}(t)^{2}}{\gamma_{d}} \\
& N(t)=v(t)^{2}+b_{1} \int_{0}^{1} e^{x} \hat{z}(x, t)^{2} d x+b_{2} \int_{0}^{1} e^{1-x} \hat{\omega}(x, t)^{2} d x \\
& V_{\text {damp }}(t)=b_{3} \int_{0}^{1} e^{x} \widetilde{\zeta}(x, t)^{2} d x+b_{4} \int_{0}^{1} e^{-x} \widetilde{\omega}(x, t)^{2} d x
\end{aligned}
$$

The form of $V_{\text {estim }}(t)$ is related to the form of the adaptive law (8) (9) and often used in adaptive control theory [6]. Applying integration by parts and (44)-(48), we compute

$$
\begin{aligned}
& \dot{V}_{\text {estim }} \leqslant \frac{1}{1+N(t)}\left(-2 c_{0} a v(t)^{2}+2 a[v(t) \hat{z}(0, t)+v(t) \widetilde{\zeta}(0, t)\right. \\
& \left.+v(t)^{2} \widetilde{q}(t)-v(t) \widetilde{d}(t)\right]+2 b_{1} \int_{0}^{1} e^{x} \hat{z}(x, t)\left(\dot{\hat{q}} g_{q}(x, t)+\dot{\hat{d}} g_{d}(x, t)\right. \\
& +[\widetilde{q}(t) v(t)-\widetilde{d}(t)+\widetilde{\zeta}(0, t)] h(x, t)) d x+b_{2}\left(e w(0, t)^{2}\right. \\
& \left.\left.-\|w(., t)\|^{2}+2 \dot{\hat{d}}(t) \int_{0}^{1} e^{1-x} w(x, t) d x\right)\right)-\frac{2 \widetilde{q}(t) \dot{\hat{q}}}{\gamma_{q}}-\frac{2 \widetilde{d}(t) \dot{\hat{d}}}{\gamma_{d}}
\end{aligned}
$$

Applying Young's and Cauchy-Schwarz's inequalities, one can get the existence of $M_{0}>0$ such that

$$
2 b_{1} \tilde{\zeta}(0, t) \int_{0}^{1} e^{x} h(x, t) \hat{z}(x, t) d x \leqslant \frac{b_{1}\|\hat{z}(t)\|^{2}}{2}+b_{1} M_{0} \tilde{\zeta}(0, t)^{2}
$$

then using the projector properties (2), (41)-(42), and the proof in [3], one can get the existence of positive functions $M_{1}\left(b_{1}\right)>0, M_{2}\left(b_{1}\right)>0$, and $M_{3}\left(b_{1}\right)>0$ such that, for all $b_{1}>0$ and for all $t \geqslant 0$,

$$
\begin{aligned}
& \dot{V}_{\text {estim }}(t) \leqslant \frac{1}{1+N(t)}\left(-\left(b_{1}-\frac{2 a}{c_{0}}-2 e b_{2}\right) \hat{z}(0, t)^{2}-\left(a c_{0}\right.\right. \\
& \left.-\gamma_{q} M_{1}\left(b_{1}\right)-2 b_{2} e\left(1+c_{0}+\bar{q}\right)^{2}-\gamma_{d} M_{2}\left(b_{1}\right)-\gamma_{d} b_{2} M_{3}\left(b_{1}\right)\right) v(t)^{2} \\
& -b_{2}\left(1-e^{2} \gamma_{d} b_{2} M_{3}\left(b_{1}\right)\right)\|\hat{\omega}\|^{2}-\left(\frac{b_{1}}{2}-\gamma_{q} M_{1}\left(b_{1}\right)-\gamma_{d} M_{2}\left(b_{1}\right)\right. \\
& \left.\left.-e \gamma_{d} b_{2} M_{3}\left(b_{1}\right)\right)\|\hat{z}\|^{2}+\left(\frac{2 a}{c_{0}}+M_{0} b_{1}\right) \widetilde{\zeta}(0, t)^{2}\right)
\end{aligned}
$$

Applying (33), integrations by parts and Young's and Cauchy-Schwarz's inequalities, one gets the existence of strictly positive constants $M_{4}, M_{5}, \varepsilon_{1}$, and $\varepsilon_{2}$ such that

$$
\begin{aligned}
& \dot{V}_{\text {damp }}(t) \leqslant b_{3}\left(-\widetilde{\zeta}(0, t)^{2}-(1+2 \lambda)\|\widetilde{\zeta}\|^{2}+\lambda M_{4}\left(\frac{\|\widetilde{\zeta}\|^{2}}{\varepsilon_{1}}\right.\right. \\
& \left.\left.+\varepsilon_{1}\left(\|\hat{z}\|^{2}+v(t)^{2}+\|\hat{\omega}\|^{2}+\|\widetilde{\omega}\|^{2}\right)\right)\right)+b_{4}\left(\widetilde{\omega}(0, t)^{2}-\frac{1+2 \lambda}{e}\right. \\
& \left.\times\|\widetilde{\omega}\|^{2}+\lambda M_{5}\left(\frac{\|\widetilde{\omega}\|^{2}}{\varepsilon_{2}}+\varepsilon_{2}\left(\|\hat{z}\|^{2}+v(t)^{2}+\|\hat{\omega}\|^{2}+\|\widetilde{\zeta}\|^{2}\right)\right)\right)
\end{aligned}
$$

Matching (57) with (58), we create a list of sufficient conditions. As the obtained bound on $\dot{V}(t)$ implies seven norms, we get seven conditions (corresponding to the fact that each factory term is chosen negative). Using that $1+$ $N(t) \leqslant e^{V(t)}$, the condition for each term is as follows ${ }^{1}$

\footnotetext{
${ }^{1}$ We have take the nomenclature as : "variable norm" ":" "condition on the associated factory term"
} 


$$
\begin{aligned}
\hat{z}(0, t)^{2}: & b_{1}>\frac{2 a}{c_{0}}+2 e b_{2} \\
\widetilde{\zeta}(0, t)^{2}: & b_{3}>b_{4}+\frac{2 a+b_{1} M_{0}}{c_{0}} \\
\|\widetilde{\zeta}(t)\|^{2}: & b_{3}(1+2 \lambda)>\lambda\left(\frac{b_{3} M_{4}}{\varepsilon_{1}}+b_{4} M_{5} \varepsilon_{2}\right) \\
\|\widetilde{\omega}(t)\|^{2}: & b_{4}\left(\frac{1}{e}+\frac{2 \lambda}{e}\right)>\lambda\left(b_{3} M_{4} \varepsilon_{1}+\frac{b_{4} M_{5}}{\varepsilon_{2}}\right) \\
v(t)^{2}: & a c_{0}>2 e b_{2}\left(1+c_{0}+\bar{q}\right)^{2} \\
& \quad+e^{V(t)} \lambda\left(b_{3} M_{4} \varepsilon_{1}+b_{4} M_{5} \varepsilon_{2}\right) \\
\|\hat{\omega}(t)\|^{2}: & e b_{2}>e^{V(t)} \lambda\left(b_{3} M_{4} \varepsilon_{1}+b_{4} M_{5} \varepsilon_{2}\right) \\
\|\hat{z}(t)\|^{2}: & b_{1}>e^{V(t)} \lambda\left(b_{3} M_{4} \varepsilon_{1}+b_{4} M_{5} \varepsilon_{2}\right)
\end{aligned}
$$

In what follows, a method for the choice of parameters condition on parameters is provided. Denoting $\widetilde{M}=\max \left(M_{4}, M_{5}\right), \lambda$ is chosen such that

$$
\lambda=\varepsilon \frac{a c_{0}}{\left(2 e\left(1+c_{0}+\bar{q}\right)^{2}+1\right) e^{V(0)} \widetilde{M}\left(b_{3} \varepsilon_{1}+b_{4} \varepsilon_{2}\right)}
$$

in which $0<\varepsilon<1$. Then $b_{2}$ is chosen such that (63)-(64) hold (which exists according to (66)). Further $b_{1}$ is chosen such that (59) holds and so does (65). Furthermore $b_{3}$ and $b_{4}$ are chosen such that conditions (60), (61) and (62) hold (a compromise needs to be chosen for $\varepsilon_{1}$ and $\varepsilon_{2}$ ). Next by choosing:

$$
\gamma_{d}+\gamma_{q}<\frac{\min \left[a c_{0}-2 b_{2}\left(1+c_{0}+\bar{q}\right)^{2}-M_{\lambda}, \frac{b_{2}}{e}-M_{\lambda}, \frac{b_{1}}{2}-M_{\lambda}\right]}{3 \max \left[M_{1}\left(b_{1}\right), M_{2}\left(b_{1}\right), b_{2} M_{3}\left(b_{1}\right)\right]}
$$

in which $M_{\lambda}=\lambda \widetilde{M} e^{V(0)}\left(b_{3} \varepsilon_{1}+b_{4} \varepsilon_{2}\right)$, using Proposition $1^{2}$ (cf. Appendix $\mathrm{C}$ ), there exists $\eta>0$, depending on initial condition $V(0)$, such that

$$
\begin{aligned}
\dot{V}(t) & \leqslant-\frac{\eta}{1+N(t)}\left(v(t)^{2}+\|\hat{z}\|^{2}+\|\hat{\omega}\|^{2}\right. \\
& \left.+\widetilde{\zeta}(0, t)^{2}+\|\widetilde{\zeta}\|^{2}+\|\widetilde{\omega}\|^{2}\right)
\end{aligned}
$$

and finally

$$
V(t) \leqslant V(0), \quad t \geqslant 0
$$

\section{E. Stability in terms of the functional $\Gamma$}

We need to establish the stability in terms of $\Gamma(t)$, i.e (12). Up to now the stability in terms of the reformulated variables $\hat{\zeta}(x, t), \hat{\omega}(x, t), \widetilde{\zeta}(x, t)$ and $\hat{\omega}(x, t)$ hold by the Lyapunov analysis, but we need to prove that it holds for (3)(5) extended by the dynamics of the unknown parameter, i.e $\hat{q}$ and $\hat{d}$. First, from the definition of the Riemann variables, and the intermediate variables, one gets

$$
u_{t}(x, t)=\frac{\hat{\zeta}(x, t)+\hat{\omega}(x, t)+\widetilde{\zeta}(x, t)+\widetilde{\omega}(x, t)}{2}
$$

one can get a similar form for $u_{x}(x, t)-\hat{d}(t)$. Therefore, applying Young's and Cauchy-Schwarz's inequalities, one

\footnotetext{
${ }^{2}$ In Proposition 1 we used $p=3 x_{1,2,3}=(v(t),\|\hat{\omega}\|,\|\hat{z}\|), m=7$ and $x_{i=4, . ., 7}$ as the remaining terms
}

can get

$$
\begin{aligned}
& \left\|u_{t}(t)\right\|^{2} \leqslant\|\hat{\zeta}(t)\|^{2}+\|\hat{\omega}(t)\|^{2}+\|\widetilde{\zeta}(t)\|^{2}+\|\widetilde{\omega}(t)\|^{2} \\
& \left\|u_{x}(t)-d\right\|^{2} \leqslant \frac{3}{2}\left(\|\hat{\zeta}(t)\|^{2}+\|\hat{\omega}(t)\|^{2}+\|\widetilde{\zeta}(t)\|^{2}+\|\widetilde{\omega}(t)\|^{2}\right)+3 \widetilde{d}(t)^{2}
\end{aligned}
$$

Second, from the backstepping transformation and its inverse, applying Young's and Cauchy-Schwarz's inequalities, one can show that there exist positive constants $r_{1}, r_{2}, s_{1}$ and $s_{2}$ such that

$$
\begin{gathered}
\|\hat{\zeta}(t)\|^{2} \leqslant r_{1} y(t)^{2}+r_{2}\|\hat{z}(t)\|^{2} \\
\|\hat{z}(t)\|^{2} \leqslant s_{1} y(t)^{2}+s_{2}\|\hat{\zeta}(t)\|^{2}
\end{gathered}
$$

Consequently, with the previous inequalities, it follows that

$$
\left\|u_{t}\right\|^{2}+\left\|u_{x}-d\right\|^{2} \leqslant\left(\frac{5}{2}\left(1+r_{1}+r_{2}\right)+3 \gamma_{d}\right)\left(e^{V(t)}-1\right)
$$

and also that

$$
\begin{gathered}
y(t)^{2} \leqslant e^{V(t)}-1 \\
\widetilde{d}(t)^{2}+\widetilde{q}(t)^{2} \leqslant\left(\gamma_{d}+\gamma_{q}\right) V(t)
\end{gathered}
$$

Therefore, we have that, for all $t \geqslant 0$

$$
\Gamma(t) \leqslant\left(1+\frac{5}{2}\left(1+r_{1}+r_{2}\right)+\gamma_{q}+4 \gamma_{d}\right)\left(e^{V(t)}-1\right)
$$

Finally, with (71) one gets

$$
\begin{aligned}
V(t) & \leqslant\left(1+e b_{1} s_{1}+\frac{1}{\gamma_{d}}+\frac{1}{\gamma_{q}}\right) \Gamma(t)+e s_{2} b_{1}\|\hat{\zeta}(t)\|^{2} \\
& +b_{2}\|\hat{\omega}(t)\|^{2}+e b_{3}\|\widetilde{\zeta}(t)\|^{2}+b_{4}\|\widetilde{\omega}(t)\|^{2}
\end{aligned}
$$

By the definition of $\widetilde{\zeta}(x, t), \widetilde{\omega}(x, t), \hat{\zeta}(x, t)$ and $\hat{\omega}(x, t)$, using Young's and Canchy-Schwarz's inequalities one gets that there exists $\rho>0$ such that

$$
V(t) \leqslant \rho \max _{s \in[0,2]} \Gamma(t-s), \quad \forall t \geqslant 0
$$

Matching the previous inequalities gives the stability result in terms of the functional $\Gamma(t)(13)$

Remark 1: One easily gets that $e^{V(0)} \leqslant e^{\rho \max _{s \in[0,2]} \Gamma(-s)}$ and so $\lambda^{*}$ can be chosen according to (66) but expressed in terms of $\max _{s \in[0,2]} \Gamma(-s)$

\section{F. Convergence analysis}

From (68), one can easily get that $N(t), \widetilde{q}(t), \widetilde{d}(t)$, and $V_{\text {damp }}(t)$ are uniformly bounded for $t \geqslant 0$, and therefore $v(t),\|\hat{z}(t)\|,\|\hat{\omega}(t)\|,\|\widetilde{\zeta}(t)\|$, and $\|\widetilde{\omega}(t)\|$ are also uniformly bounded for $t \geqslant 0$. Consequently, from (70), $\|\hat{\zeta}(t)\|$ is also bounded for $t \geqslant 0$.

From there, applying Young's inequality to (8) and (9), one can obtain that $\dot{\hat{q}}(t)$ and $\dot{\hat{d}}(t)$ are uniformly bounded for $t \geqslant 0$. Similarly, applying Cauchy-Schwarz's inequality to (6), one can obtain that $\hat{\zeta}(1, t)$ is uniformly bounded for $t \geqslant 0$. Using (25)-(26) one obtains that $\widetilde{\zeta}(x, t)$ is uniformly bounded for $t \geqslant 1$ and in particular $\widetilde{\zeta}(0, t)$ is uniformly bounded for $t \geqslant 1$. Moreover, as $\hat{\zeta}(x, t)=\hat{\zeta}(1, t-1+x)-\hat{d}(t)+\hat{d}(t-1+x)$, $\hat{\zeta}(x, t)$ is also uniformly bounded for $t \geqslant 1-x$ and in particular $\hat{\zeta}(0, t)$ is uniformly bounded for $t \geqslant 1$. From (40), $\hat{z}(0, t)=\hat{\zeta}(0, t)+\left(c_{0}+\hat{q}(t)-1\right) y(t)$, and recall $\widetilde{\zeta}(1, t)=0$, 
consequently, $\hat{z}(0, t)$ is also uniformly bounded for $t \geqslant 1$. Further, from (44)-(48) and (33),

$$
\begin{aligned}
& \frac{d}{d t} v(t)^{2}=2 a v(t)\left(-c_{0} y(t)+\hat{z}(0, t)+\widetilde{\zeta}(0, t)+v(t) \widetilde{q}(t)-\widetilde{d}(t)\right) \\
& \frac{d}{d t}\|\hat{z}(t)\|^{2}=-\hat{z}(0, t)^{2}+2 \int_{0}^{1} \hat{z}(x, t)\left(g_{q}(x, t) \dot{\hat{q}}(t)\right. \\
& \left.+\dot{\hat{d}}_{d}(x, t)+[\widetilde{q}(t) v(t)+\widetilde{d}(t)] h(x, t)\right) d x \\
& \frac{d}{d t}\|\hat{\omega}(t)\|^{2}=\hat{\omega}(1, t)^{2}-\hat{\omega}(0, t)^{2}+2 \dot{\hat{d}(t)} \int_{0}^{1} \hat{\omega}(x, t) d x
\end{aligned}
$$

in which $\hat{\omega}(0, t)=\left(1+c_{0}+\hat{q}(t)\right) y(t)-\hat{\zeta}(0, t)$. Moreover, as $\hat{\omega}(1, t)=\hat{\omega}(0, t)+\hat{d}(t)-\hat{d}(t-1)$, and using (49)-(51), (8), (9), Cauchy-Schwarz's inequality and the previous considerations, it follows that the right-hand terms in the previous equations are all uniformly bounded for $t \geqslant 2$. Furthermore, one gets (see appendix B)

$$
\begin{aligned}
& \left|\frac{d}{d t}\|\widetilde{\zeta}(t)\|^{2}\right| \leqslant 16 \lambda^{2} \max _{s \in[0,1]}\left\|u_{t}(t-s)\right\|^{2} \\
& \left|\frac{d}{d t}\|\widetilde{\omega}(t)\|^{2}\right| \leqslant 64 \lambda^{2} \max _{s \in[0,2]}\left\|u_{t}(t-s)\right\|^{2} .
\end{aligned}
$$

As $\left\|u_{t}(t)\right\|$ is uniformly bounded -as $\Gamma(t)$ is- the lefthand terms on the previous equations are also uniformly bounded for $t \geqslant 2$. Finally, integrating (67) from 0 to $\infty$, it follows that $y(t),\|\hat{z}(t)\|,\|\hat{\omega}(t)\|,\|\widetilde{\zeta}(t)\|$, and $\|\widetilde{\omega}(t)\|$ are square integrable. Following Barbalat's Lemma, $y(t),\|\hat{z}(t)\|$, $\|\hat{\omega}(t)\|,\|\widetilde{\zeta}(t)\|$, and $\|\widetilde{\omega}(t)\|$ tend to zero as $t$ tend to $\infty$. Using (69), it follows that $\left|u_{t}(0, t)\right|,\left\|u_{t}(t)\right\|$ and $\left\|u_{x}-\hat{d}(t)\right\|$ tend to zero as $t$ tends to $\infty$.

\section{APPLICATION AND NUMERICAL SIMULATION}

The wave equation (3)-(4) is a normalized linearized model for the stick-slip phenomenon [8] that occurs in drilling operation (e.g. [3], [4] and [16]). The drillstring angular oscillations can be modeled by a wave equation with a nonlinear boundary condition $u_{t t}(0, t)=a F\left(u_{t}(0, t)+a u_{x}(0, t)\right.$ (see [17]), accounting for the friction between the drillbit and the rock. Even if there exist phenomenological expressions of this friction $F$ [18], [17] and [14], they depend on parameters, such as the weight on the bit, drilling mud properties, and the nature of the rock. So they can change during the drilling process. This is the reason why using an adaptive controller could be of high interest for this application. In simulation, we consider the nonlinear model coupled with our control law.

The control law (6) and adaptation laws (8)-(10) are not used for stabilization but for regulation, i.e., we need to control $u_{t}(x, t)-u_{t}^{\text {ref }}$ instead of $u_{t}(x, t)$. We choose $c_{0}$ such that $a c_{0}=1, b_{2}=10^{-4}, b_{1}=1, \gamma_{d}=0.5$ and $\gamma_{q}=0.01$. Simulations are performed using physical variables, which are listed with their used corresponding values in Table 1 and taken from [16] and [7]. Relation between this simulation model and the normalized one is explained in [16]. The reference, $u_{t}^{\text {ref }}$ is taken such that the unnormalized velocity is $\theta_{t}\left(\xi, c_{t} t\right)=5[\mathrm{rad} / \mathrm{s}]$, in which $c_{t}$ is the time normalization

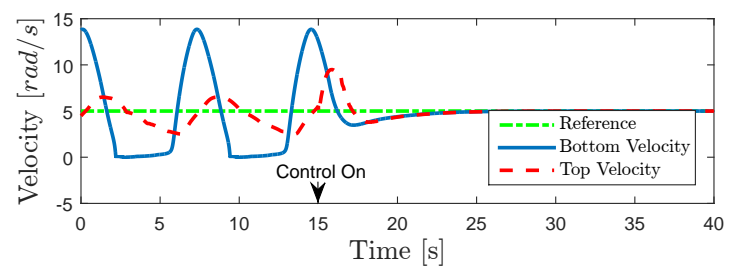

Fig. 1. Simulation of the top and bottom velocities. The adaptive controller, i.e (6), (8) and (9) is turned on at $15 \mathrm{sec}$. The top and bottom velocities converge at $95 \%$ of the reference, at respectively 22.5 and $22.3 \mathrm{sec}$ corresponding to settling times equal to 7.5 and $7.3 \mathrm{sec}$.
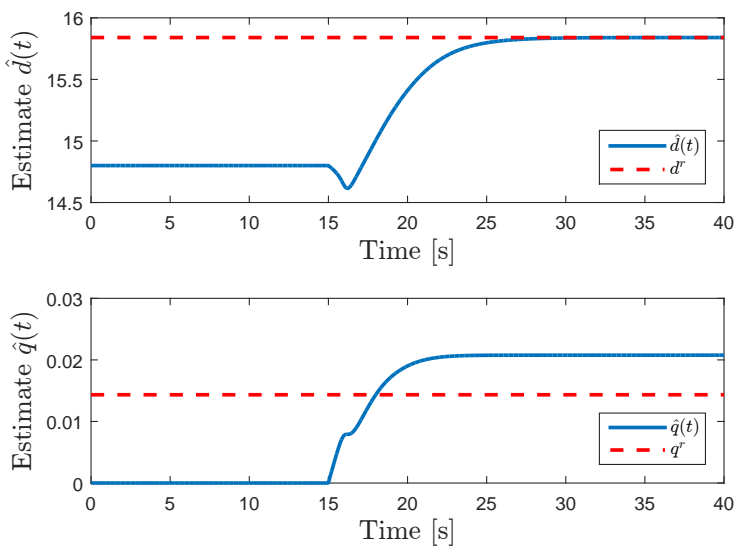

Fig. 2. Simulation of the parameter estimations. Even if velocity regulation is obtained, one can observe that the estimation $\hat{q}(t)$ does not converge to the value of $q$. Note that this latter observation does not contradict the conclusion of Theorem 1. Finally, as expected, $\hat{d}(t)$ converges to $d$.

coefficient. The top and bottom velocities are displayed in Figure 1, and the parameter estimations of $d$ and $q$ are displayed in Figure 2. One can observe that the oscillation existing in the open-loop phase, i.e. before $15 \mathrm{sec}$, is compensated for by the application of the control law.

\section{CONCLUSION}

Simulations have illustrated the practical relevance of the proposed method for the regulation of a "stick-slip"

\begin{tabular}{|c|c|c|}
\hline Symbol & Description & Value \\
\hline $\bar{L}$ & Length of the drillstring & $2000[\mathrm{~m}]$ \\
\hline$J$ & Drillstring second moment of area & $1.19 \mathrm{e}-5\left[\mathrm{~m}^{4}\right]$ \\
\hline$G$ & Shear modulo & $79.3 \mathrm{e} 10\left[\mathrm{~N} / \mathrm{m}^{2}\right]$ \\
\hline$I$ & Drillstring inertia's moment per length's unit & $9.5 \mathrm{e}-3[\mathrm{~kg} . \mathrm{m}]$ \\
\hline$I_{b}$ & BHA moment of inertia & $311\left[\mathrm{~kg} . \mathrm{m}^{2}\right]$ \\
\hline$D_{d p}$ & Outer diameter of the drill pipe & $1.08 \mathrm{e}-1[\mathrm{~m}]$ \\
\hline$\beta$ & Viscous in-domain damping coefficient & $0.05[1 / s]$ \\
\hline$c_{b}$ & Sliding torque coefficient & 2.e3 $[$ N.m.s/rad $]$ \\
\hline$T_{t o b}$ & Torque on the bit parameter & $7.5 \mathrm{e} 2[N . m]$ \\
\hline$\alpha_{1}, \alpha_{2}, \alpha_{3}$ & Friction parameters & $5.5 ; 2.2 ; 3500$ \\
\hline$\gamma$ & Damping parameter & $0.03[$ N.m.s $/ \mathrm{rad}]$ \\
\hline
\end{tabular}
phenomenon in oil-drilling, but it can be applied to a much larger class of physical problems as it works for unknown dynamics boundary. A limit of the present method is that we consider in-domain damping as a disturbance (even if in-domain damping may actually help the stabilization). For

TABLE I

PARAMETERS VALUES 
this reason, future works will focus on developing control laws tailored to handle in-domain damping.

\section{APPENDIX}

\section{A. PHYSICAL MODEL AND NORMALIZATION}

Following [17], denoted $\theta(\xi, \tau)$ the angle of rotation of the drill string at $\xi$ length and time $\tau$. By convention the top boundary is at $\xi=0$ and the bottom boundary at $\xi=L$, the torsional dynamics can be modeled by

$$
G J \theta_{\xi \xi}(\xi, \tau)-I \theta_{\tau \tau}(\xi, \tau)-\beta \theta_{\tau}(\xi, \tau)=0
$$

with the following boundary condition

$$
\begin{aligned}
& G J \theta_{\xi}(0, \tau)=c_{\alpha}\left(\theta_{\tau}(0, \tau)-\Omega(\tau)\right) \\
& I_{b} \theta_{\tau \tau}(L, \tau)=-G J \theta_{\xi}(L, \tau)-T_{B I T}\left(\theta_{t}(L, \tau)\right)
\end{aligned}
$$

in which $\Omega(\tau)$ is the angular velocity of the rotatory table rotor, $T_{B I T}($.$) is the non-linear rock-on-bit friction term and$ other constants are listed in Table I. Following [16], we consider the changes of variables:

$$
\begin{aligned}
\xi & =L(1-x) \\
\tau & =L \sqrt{\frac{I}{G J}} t \triangleq c_{t} t \\
u(x, t) & =\theta(\xi, \tau) \\
U(t) & =\frac{L c_{\alpha}}{G J}\left(\Omega\left(c_{t} t\right)-\frac{1}{c_{t}} u_{t}(1, t)\right) \\
F(\chi) & =-\frac{L}{G J} T_{B I T}\left(\frac{\chi}{c_{t}}\right)
\end{aligned}
$$

with the following constant

$$
a=L \frac{I}{I_{B}}, \quad \lambda=\beta L \sqrt{\frac{1}{G J L}}
$$

and consider (82) around an equilibrium $u_{t}^{r}$, i.e,

$$
F\left(u_{t}(0, t)\right)=q\left(u_{t}(0, t)-u_{t}^{r}\right)-d
$$

in which $d=-F\left(u_{t}^{r}\right)$ and $q=\frac{\partial F}{\partial w}\left(u_{t}^{r}\right)$. Then assuming $u_{t}^{r}=$ 0 for the sake of the equation conciseness, the physical torsional dynamics model can be reformulated under the from (3)-(5). For the simulation we consider the friction term $T_{B I T}$ (.) has been taken from [18], that is

$$
T_{B I T}(\chi)=\gamma \chi+\frac{2 T_{t o b}}{\pi}\left(\alpha_{1} \chi e^{-\alpha_{2}|\chi|}+\arctan \left(\alpha_{3} \chi\right)\right)
$$

the used parameter values are gathering in Table I. If we consider the drill pipe to be in contact of a viscous milieu, denoting $\mu$ the viscous coefficient of the milieu, then the associated damping coefficient is equal to:

$$
\beta=\mu \pi L \frac{D_{d p}^{2}}{2}
$$

\section{B. Proof of (73)-(74)}

$$
\begin{aligned}
& \frac{d}{d t}\|\widetilde{\zeta}(t)\|^{2}=4 \lambda^{2} \frac{d}{d t} \int_{0}^{1}\left\{\int_{x}^{1} u_{t}(\chi, t+x-\chi) d \chi\right\}^{2} d x \\
& =8 \lambda^{2} \int_{0}^{1} \int_{x}^{1} u_{t t}(\chi, t+x-\chi) d \chi \int_{x}^{1} u_{t}(\chi, t+x-\chi) d \chi d x
\end{aligned}
$$

or applying Cauchy-Schwarz's inequality

$$
\left|\int_{x}^{1} u_{t}(\chi, t+x-\chi) d \chi\right| \leqslant \max _{s \in[0,1]}\left\|u_{t}(t-s)\right\|
$$

and applying Fubini's theorem

$$
\begin{aligned}
\mid \int_{0}^{1} \int_{x}^{1} u_{t t}(\chi, t+ & x-\chi) d \chi d x \mid \\
& =\left|\int_{0}^{1} \int_{0}^{\chi} u_{t t}(\chi, t+x-\chi) d x d \chi\right| \\
& =\left|\int_{0}^{1}\left(u_{t}(\chi, t)-u_{t}(\chi, t-\chi)\right) d \chi\right| \\
& \leqslant 2 \max _{s \in[0,1]}\left\|u_{t}(t-s)\right\|
\end{aligned}
$$

thus

$$
\left|\frac{d}{d t}\|\widetilde{\zeta}(t)\|^{2}\right| \leqslant 16 \lambda^{2} \max _{s \in[0,1]}\left\|u_{t}(t-s)\right\|
$$

Similarly, one gets

$$
\left|\frac{d}{d t}\|\widetilde{\omega}(t)\|^{2}\right| \leqslant 64 \lambda^{2} \max _{s \in[0,2]}\left\|u_{t}(t-s)\right\|^{2}
$$

\section{Technical result}

Proposition 1: Consider $x_{i}(t) \in L_{2}, \forall 0<i \leqslant p+m$, where $p \leqslant m \in \mathbb{N}^{*}, V(t)$ is a positive definite functional of $\left(x_{i}(t)^{2}\right)_{i=1 \ldots m}$ assume there exists a $K_{\infty}$ function denoted $K($. such that

$$
\dot{V}(t) \leqslant-\sum_{i=1}^{p}\left(a_{i}-K(V(t)) x_{i}(t)^{2}-\sum_{i=p+1}^{m} b_{i} x_{i}(t)^{2}, \forall t \geqslant 0\right.
$$

in which $a_{i}>0, \quad b_{i}>0 \quad \forall i$ if $a_{i}-K(V(0))>0, \forall i$ then $a_{i}-K(V(t))>0, \forall i \forall t \geqslant 0$

Proof: For the sake of simplicity, take $p=1$ (similar arguments hold for the general case). By contradiction, assume the existence of $t_{0}$ such that $a_{1}-K\left(V\left(t_{0}\right)\right) \leqslant 0$, $K(V(t))$ being continuous using Bolzano's theorem, there exists $t_{1} \in\left[0, t_{0}\right]$ such that $a-K\left(V\left(t_{1}\right)\right)=0$. We consider $t_{2}=$ inf $\left\{t_{1} \geqslant 0 / a=K\left(V\left(t_{1}\right)\right)\right\}$. Using the mean value theorem , there exists $t_{3} \in\left[0, t_{2}\right]$ such that $\frac{K(V(t))}{d t}\left(t_{3}\right)>0$. Further using the fact that $K($.$) is K_{\infty}$ and so does $K(.)^{-1}$, we have $\dot{V}\left(t_{3}\right)>0$. We arrive to a contraction with (87) for $t \in\left[0, t_{3}\right]$.

\section{REFERENCES}

[1] A. M. Annaswamy and A. F. Ghoniem. Active control of combustion instability: Theory and practice. Control Systems, IEEE, 22(6):37-54, 2002.

[2] Z. Artstein. Linear systems with delayed controls: a reduction. IEEE Transactions on Automatic Control, 27(4):869-879, 1982. 
[3] D. Bresch-Pietri and M. Krstic. Adaptive output feedback for oil drilling stick-slip instability modeled by wave PDE with anti-damped dynamic boundary. American Control Conference (ACC), Portland, $O R$, pages 386-391, June 2014.

[4] D. Bresch-Pietri and M. Krstic. Adaptive output-feedback for wave PDE with anti-damping -Application to surface-based control of oil drilling stick-slip instability. In 53rd Conference on Decision and Control, Los Angeles, CA, pages 1295-1300, 2014.

[5] W. M. Haynes. CRC handbook of chemistry and physics. CRC press, 2014.

[6] P. A. Ioannou and J. Sun. Robust Adaptive Control. Prentice Hall, 1996.

[7] J. Jansen. Nonlinear dynamics of oilwell drillstrings. $\mathrm{PhD}$ thesis, Delft University of Technology, 1993.

[8] J. Jansen and L. van der Steen. Active damping of self-excited torsional vibrations in oil well drillstrings. Journal of Sound and Vibration, pages 179(4):647-688, 1995

[9] M. Krstic. Dead-time compensation for wave/string PDEs. Journal of Dynamic Systems, Measurement, and Control, 133(3):031004, 2011.

[10] M. Krstic and A. Smysklyaev. Boundary Control of PDEs. SIAM Advances in Design and Control, 2008.

[11] W. Kwon and A. Pearson. Feedback stabilization of linear systems with delayed control. IEEE Transactions on Automatic Control, 25(2):266-269, 1980.

[12] A. Manitius and A. W. Olbrot. Finite spectrum assignment problem for systems with delays. IEEE Transactions on Automatic Control, 24(4):541-552, 1979.

[13] M. S. D. Queiroz and C. D. Rahn. Boundary Control of Vibration and Noise in Distributed Parameter Systems: An Overview. Mechanical Systems and Signal Processing, 16(1):19 - 38, 2002.

[14] T. Ritto, C. Soize, and R. Sampaio. Non-linear dynamics of a drillstring with uncertain model of the bit-rock interaction. International Journal of Non-Linear Mechanics, pages 44(8):865-876, 2009.

[15] P. Rouchon. Flatness and stick-slip oscillation. Technical report, MINES ParisTech, 1998.

[16] C. Sagert, F. Di Meglio, M. Krstic, and P. Rouchon. Backstepping and flatness approaches for stabilization of the stick-slip phenomenon for drilling. IFAC Sympositium on System Structure and Control, Grenoble, France, pages 779-784, 2013.

[17] M. B. Saldivar, S. Mondie, J. J. Loiseau, and V. Rasvan. Stick-Slip Oscillations in Oillwell Drillstrings: Distributed Parameter and Neutral Type Retarded Model Approaches. In Proceeding of the 18th IFAC World Congress, Milano, Italy, pages 18:284-289, 2011.

[18] Q.-Z. Zhang, Y.-Y. He, L. Li, et al. Sliding mode control of rotatory drilling system with stick slip oscillations. In 2nd International Workshop on Intelligent Systems and Applications (ISA), Wuhan, China, pages 1-4, 2010. 